\title{
EVALUATING ATTITUDES ON THE QUALITY OF SERVICE OF PEDESTRIAN NETWORKS
}

\author{
GEORGE PAPAGEORGIOU, ATHANASIOS MAIMARIS, THEOFANO EFSTATHIADOU \\ \& EUDOKIA BALAMOU \\ E.U.C. Research Center, Nicosia, Cyprus
}

\begin{abstract}
The aim of our research is to study opinions of people upon the pedestrian conditions in urban spaces. The goal is to evaluate, based on the perception of citizens, the existence of certain walkability factors that contribute to the quality of service of the pedestrian environment. Walkability factors are related to mobility, connectivity, comfort, safety and convenience. As a result, a pedestrian satisfaction index could be derived, which might reveal the citizens' quality of life and the need for developing comfortable, healthy and safe pedestrian networks. Results show the willingness of people to walk more, but also the obstacles that prevent them from doing so. Our study is important for the better understanding of walkability issues in urban spaces, especially for developing technology solutions and strategies for local governments in the quest of realizing the vision of the smart city.

Keywords: urban development, pedestrian networks, walkability, quality of service.
\end{abstract}

\section{INTRODUCTION}

Walking is the healthiest, cheapest and the most universal mode of transportation. With today's hectic way of life, people spend most of their time indoors and they seldom go for walks, which would be good exercise and beneficial for their health. In a ddition, walking could be an important transportation mode, which is good for the natural environment since it has zero carbon footprint. Walking also positively contributes to socioeconomic development for any country. Therefore, a walkable urban environment is necessary for sustainable development.

As a result, walkability gained attention in recent years. This is where the walkability concept plays a significant role. By effectively assessing walkability, areas of improvement of the pedestrian network can be identified. F urther, w ith t oday's I nternet of Things (IoT) technology, the opportunity is created where citizens together with city planning professionals and local government officials may be able to jointly to carry out in order to improve the quality of the pedestrian environment, supporting more objective, effective and comprehensive walking-related strategies and interventions.

This paper presents the results of an initial survey, for the purpose of examining citizens' attitudes on walkability and factors that influence the decision to walk. Based on a literature review, a questionnaire was prepared which involved walking habits, aspects of the pedestrian environment and their influence on walkability as well as the potential of information and communication technology. The collected data was further analyzed, the results of which are presented in this paper.

\section{LITERATURE REVIEW}

Walkability recently gained a lot of interest from researchers. A range of methodologies (qualitative and quantitative) exist in the current literature that assess the pedestrian environment and give an insight on the attitudes of citizens regarding the factors that affect walkability. In this section, we present a review of the most important studies related to walkability and citizen satisfaction. 
In a study carried out in Ghent, Belgium [1], we see that the relationship between objectively assessed walkability characteristics and neighborhood satisfaction is a complex topic. The adults living in a higher walkable neighborhood were less satisfied as residential density is negatively related to neighborhood satisfaction. Also, the study revealed that there is no association between satisfaction and street connectivity or land use mix. This study shows that even though high walkability is desirable, it might also bring negative consequences on the quality of life. Therefore, there is a need to analyze walkability so that we derive the real factors that promote a positive impact to all stakeholders involved.

In another study carried out by De Silva et al. [2] it was attempted to understand how environmental factors affect University students' perceptions of safety, which were quantified based on their arousal, i.e. the calmness or stress felt, and walking speeds. The study was able to rank 10 identified environmental cues based on the extent to which each contributes towards the perception for safety, during both the day and night times. The study showed that people are more likely to feel unsafe in areas with low degree of visibility and low levels of physical upkeep during the day. Further, it was found that people most likely felt unsafe in areas, where there were few land uses that attracted people and in areas where households were not amply distributed. The results also indicated that people, both men and women, have a tendency of walking faster in areas demarcated as unsafe and the walking speeds of people during the night are much faster than that of the day.

Notable for assessing walkability of the pedestrian environment is the research project of Kelly et al. [3] that was designed to increase the understanding of the factors that influence the levels of walking and pedestrian route choice. The study describes a number of techniques that were used to assess the pedestrian environment from a pedestrian's perspective. As a result, a computer based tool developed using stated preference surveys to determine the relative values of a range of factors in the pedestrian environment; an onstreet survey was designed to investigate values and attitudes towards different attributes of the pedestrian environment along a route; and an 'on the move survey' was carried out, where pedestrian volunteers were interviewed while walking along the route in order to get an actual account of their experiences as they walk. The results indicated that there were a number of pedestrian attributes considered important by pedestrians when walking including pavement cleanliness, safe crossing places, good connectivity and a sense of security. Also, results showed that the walking experience is affected by the cumulative impact of multiple interactions (both positive and negative) as people walk in the pedestrian environment. Furthermore, it highlighted the importance of traffic volume to pedestrians and identified that clean pavements, connectivity and a perception of safety are some of the factors which improve the quality pedestrian environment.

Yet another study regarding walkability, conducted by Zainol et al. [4] in Georgetown, Penang, a UNESCO World Heritage Site, aimed to assess the physical attributes of pedestrianization in the city and to examine citizens' satisfaction on the pedestrian facilities using six walkability features including a) pathway, b) zebra crossing, c) signage, d) personal safety, e) traffic flow and, f) aesthetics and amenities. Relative Importance Index (RII) analysis showed that existing pathways do not meet acceptable standards in promoting a walkable environment. Aesthetic and amenities scores the lowest in satisfactory level and zebra crossing scores the highest in unsatisfactory level. Finally, the study showed that the level of walkability in the city was found to be inadequate. Further, the provision of physical walking and built heritage features without providing sufficient activities along the streets do not yield high walkability.

The work of Moura et al. [5] presents a participatory framework for the assessment of walkability based on local circumstances and expertise, replicable on distinct urban contexts. 
The framework takes into account distinct pedestrian groups (adults, children, seniors and impaired mobility pedestrians) and trip purposes (utilitarian, leisure), expressing walkability in terms of seven key dimensions (7C's layout, Connected, Convenient, Comfortable, Convivial, Conspicuous, Coexistence, Commitment). It was applied to an area in central Lisbon, Portugal, in order to evaluate the ease or difficulty that different types of pedestrians can face in their walking activities and, potentially, providing an insight for intervention and improvements. The results showed clear differences in walkability scores for different pedestrian groups, namely between adults and seniors or impaired pedestrians. Besides, a validation of the results is presented by comparing street performance, as measured by homebased surveys conducted within the study area. Validated results confirmed that the evaluation framework proposed is reliable in the representation of the pedestrian environment qualities as perceived by the public.

Alternatively, Kim et al. [6] investigated the relationships between pedestrian satisfaction and a variety of built environment factors, in order to gain insight into urban design strategies that can improve both pedestrian satisfaction and activities. Using econometric instruments, the authors showed that there is significant correlation between pedestrian satisfaction and built environmental factors indicating the importance of considering psychological factors for walkability studies, such as different pedestrian groups and trip motives. Gebel et al. [7] followed similar approaches in order to check whether persons who perceive their objectively measured high-walkable environment as low-walkable. This contrast between objective measurements and citizens' attitudes, reveals the need for survey studies to include people preferences and context.

Furthermore, it is important to note that the existence of new technologies and the extensive use of smartphones by pedestrians creates an additional factor to consider when designing our pedestrian networks. Instead of prohibiting the use of a smartphone at crossings and other areas of the network, it is probably more wise to redesign our urban spaces to safely accommodate the use of smartphones. This is imperative as the smartphone could possess the capability of enhancing walkability.

The above studies show that walkability is a very complex topic that needs thorough analysis. People could be motivated to walk by many factors, such as connectivity, convenience, comfort, conviviality, conspicuity, coexistence, commitment, and safety. These factors influence citizens' willingness to walk at various degrees depending on the type of pedestrian group such as adults, children, seniors and impaired mobility pedestrians. Further, there are more moderating factors that influence willingness to walk, namely the trip purpose whether it is for utility, exercise or leisure. Therefore, the context, which includes the pedestrian culture, should always be taken into consideration in walkability studies, especially for the purpose of assessing the pedestrian network of a particular urban environment.

Finally, technology and intelligent systems provide new avenues for enhancing walkability. Even though Intelligent Transportation Systems (ITS) is widely used for enhancing vehicular traffic, little has been done for promoting pedestrian flows. The authors, having wide experience with ITS applications [8]- [13], see the enormous potential that exists for what they call Intelligent Pedestrian Mobility Systems (IPMS) [14].

The next section describes the methodology we have employed in order to examine some of the most important aspects of walkability, taking into consideration the above findings.

\section{METHODOLOGY}

To get an insight to the attitudes of citizens regarding the factors affecting walkability, a multi-dimensional questionnaire, with Likert-scale questions, was employed. Questionnaires 

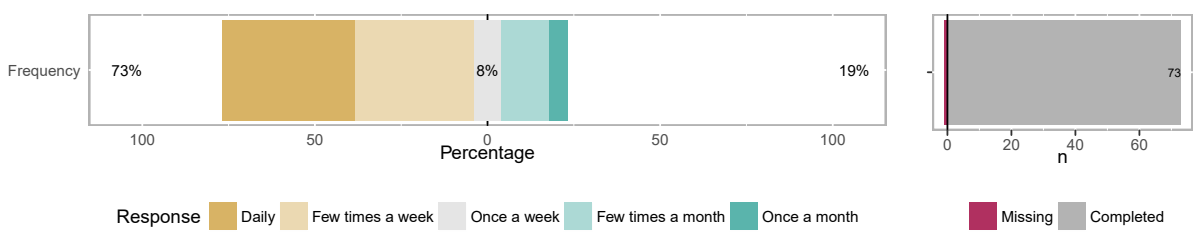

Figure 1: Frequency of walking.

were distributed to university students in Cyprus via a simple random sampling technique using clusters (cluster sampling).

The questionnaire involved firstly demographic questions about gender, age group, academic qualifications, occupation and nationality. Then, questions were asked on traveling habits regarding the mode of transportation, such as car, bus, motorbike and bicycle in relation to walking. Further, the importance citizens assign on the various walkability factors (comfort, connectivity, conviviality, conspicuity, convenience) was drawn via the survey. Questions were also asked regarding walking frequency and preferences on time of day to walk. Moreover, there were questions on possible improvements of the pedestrian network. Finally, the willingness of citizens to use a smartphone application in order to enhance their walking experience was addressed.

The questionnaire was answered by 74 respondents from various classes who were selected randomly from our sampling frame. The total number of the selected respondents was 104 which gives us a response rate of $71 \%$. We made sure that the respondents had enough time to answer the questions and clarifications were also provided when necessary in a non-leading manner. The collected data was statistically analyzed using SPSS v24 and R v.3.4.1, the results of which are presented in the next section.

\section{RESULTS}

Based on the collected data, analysis was carried out, and it is presented in this section as follows. As seen from the demographic data, a representative sample was gathered of young people, $97 \%$ of which are between 19-40 years old. Most of them were highly educated, $89 \%$ of which have a university degree at bachelor, master or $\mathrm{PhD}$ level. It is important to note that $34 \%$ of respondents had a foreign nationality, which is also representative as Nicosia is a cosmopolitan city with a large number of foreign students.

Further, an important finding that comes out of our analysis is that most of the respondents are walking. As shown in Fig. 1, 73\% declare they walk on a daily basis or at least a few times a week. Even though our sample respondents mostly use the car as a transportation means ( sample mean $=4.5 / 5$ ), at the same time walking is a favored transportation method (sample mean $=3.6 / 5$ ). Unfortunately, public transportation is not preferred (sample mean $=2.2 / 5$ ), probably due to inadequate route selection and availability. Further, there is a relatively high use of the bicycle (sample mean $=2.4 / 5$ ).

It is important to note that Comfort, Connectivity, Conviviality, Conspicuity, and Convenience are all factors being considered when deciding to walk. As shown in Fig. 2, sample means of around 4/5 show a strong agreement that pedestrian networks should have well maintained footpaths, with undisturbed routes, access to public transport, comfortable walking conditions, clear signing and information, and walkable distances. It seems that the preferred walking hours are during the afternoon and evening. This calls for adequate lighting and visibility in order to increase the level of service of the pedestrian network. 

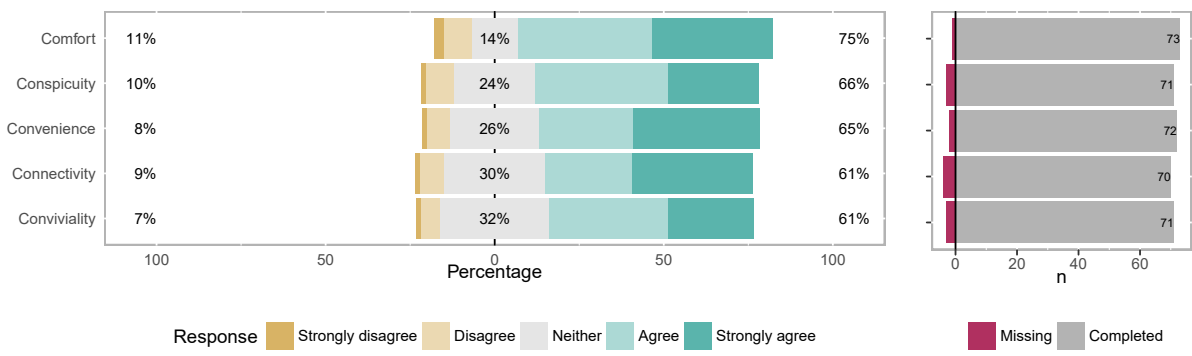

Figure 2: Factors considered when deciding to walk.
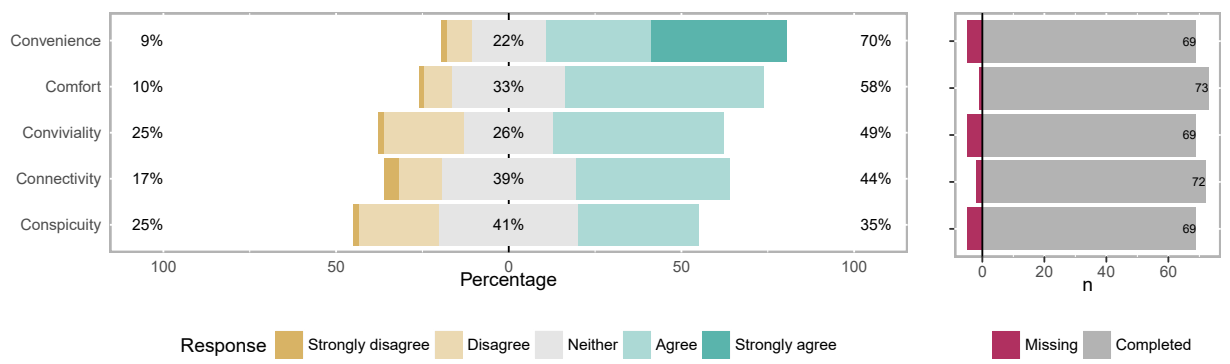

Figure 3: Areas that need improvement in the pedestrian networks.

A finding that opens up the possibility of employing new technology in our pedestrian networks is that the majority of our respondents declare that the use of an information based smartphone application would encourage them to walk more. This finding reveals the need to carry out further research in order to elicit the pedestrian requirements of such an application as well as possible co-development with the local authorities.

Another important finding is that the current situation of the level of service of the pedestrian networks in Cyprus needs a lot of improvement. As shown in Fig. 3, the respondents strongly agree on this aspect with a sample mean 4.5/5. This information should definitely be considered by the local authorities and probably involve the citizens in an extended evaluation of the pedestrian networks. Our sample respondents demand improvements on all previously examined factors (Comfort, Connectivity, Conviviality, Conspicuity, and Convenience).

To examine any possible differences of male and female respondents we performed an independent samples t-test, which showed that there is no significant difference on the choice of transport mode between male and female respondents. Further, there are no significant differences between the age groups on the choice of transport mode.

Further, correlational analysis was carried out to examine relationships among our variables. A relatively high correlation coefficient of 0.5 exists between the use of bus transport mode and walking. This shows the importance of developing a system for providing the necessary information, possibly through a smartphone application, for combining the two modes, especially for connectivity purposes.

Also, correlational analysis was carried out between factors influencing the decision to walk and areas of the pedestrian network that need improvement. Specifically, a correlation 
coefficient of 0.4 revealed to us that someone deciding to walk for reasons of convenience, at the same time demands improvements on the convenience offered by the pedestrian network.

\section{CONCLUSIONS}

This paper examined walkability aspects based on citizens' perception and attitudes. There were no differences in the opinions of male and female respondents. Especially, young people opinions were gathered on walking habits, pedestrian network infrastructure adequacy and the potential for a smartphone application to enhance the walking experience.

Factors such as comfort, connectivity, conviviality, conspicuity, and convenience are all being considered when deciding to walk. We have found a strong agreement that pedestrian networks should have well maintained footpaths, with undisturbed routes, access to public transport, comfortable walking conditions, clear signing and information, and walkable distances. Further, there are indications that the current situation of the level of service of the pedestrian networks in Cyprus needs a lot of improvement. Preferred walking hours are during the afternoon and evening, which calls for adequate lighting and visibility.

A relatively high correlation coefficient between the use of bus transport mode and walking was found, which shows the importance of developing a system for providing the necessary information in order to combine the two modes.

Finally, it was revealed that an information-based smartphone application would encourage people to walk more. There is a need to carry out further research in order to elicit the pedestrian requirements of such an application as well as possible co-development with the local authorities.

\section{ACKNOWLEDGEMENTS}

The research presented in this paper is co-funded by the Republic of Cyprus and the European Regional Development Fund as part of ERA-NET Cofund Smart Urban Futures (ENSUF) Joint Programming Initiative (JPI) Urban Europe, though the Research Promotion Foundation, protocol no. KOINA/ПKП URBAN EUROPE/1215/11. This framework is supported by the European Commission and funded under the HORIZON 2020 ERA-NET Cofund scheme.

\section{REFERENCES}

[1] Dyck, D.V., Cardon, G., Deforche, B. \& De Bourdeaudhuij, I., Do adults like living in high-walkable neighborhoods? Associations of walkability parameters with neighborhood satisfaction and possible mediators. Health and Place, 17, pp. 971-977, 2011.

[2] De Silva, C., Warusavitharana, E. \& Ratnayake, R., An examination of the temporal effects of environmental cues on pedestrians' feelings of safety. Computers, Environment and Urban Systems, 64, pp. 266-274, 2017.

[3] Kelly, C., Tight, M., Hodgson, F. \& Page, M., A comparison of three methods for assessing the walkability of the pedestrian environment. Journal of Transport Geography, 19(Special section on Alternative Travel futures), pp. 1500-1508, 2011.

[4] Zainol, R., Ahmad, F., Mohd Aripin, A.W., Chen, W., Salleh, H. \& Ali, A.S., Pedestrianization and walkability in a fast developing unesco world heritage city. Open House International, 41(1), p. 112, 2016.

[5] Moura, F., Cambra, P. \& Gonçalves, A.B., Measuring walkability for distinct pedestrian groups with a participatory assessment method: A case study in Lisbon. Landscape and Urban Planning, 157, pp. 282-296, 2017. 
[6] Kim, S., Park, S. \& Lee, J.S., Meso- or micro-scale? environmental factors influencing pedestrian satisfaction. Transportation Research Part D, 30, pp. 10-20, 2014.

[7] Gebel, K., Bauman, A.E., Sugiyama, T. \& Owen, N., Mismatch between perceived and objectively assessed neighborhood walkability attributes: Prospective relationships with walking and weight gain. Health and Place, 17(Geographies of Care), pp. 519-524, 2011.

[8] Maimaris, A. \& Papageorgiou, G., A review of intelligent transportation systems from a communications technology perspective. 2016 IEEE 19th International Conference on Intelligent Transportation Systems (ITSC), pp. 54-59, 2016.

[9] Papageorgiou, G., Maimaris, A. \& Ioannou, P., Analysis and evaluation of intelligent bus rapid transit systems in Cyprus. 2015 IEEE 18th International Conference on Intelligent Transportation Systems (ITSC), IEEE, pp. 95-100, 2015.

[10] Papageorgiou, G., Maimaris, A., Ioannou, P. \& Aphamis, T., Planning for effective bus rapid transit systems: A scenario simulation modelling based approach. 13th IFAC Symposium on Control in Transportation Systems (CTS'2012), pp. 366-371, 2012.

[11] Papageorgiou, G., Ioannou, P., Pitsillides, A., Aphamis, T. \& Maimaris, A., Development and evaluation of bus priority scenarios via microscopic simulation models. 12th IFAC Symposium on Transportation Systems (CTS'09), pp. 434-441, 2009.

[12] Papageorgiou, G., Damianou, P., Pitsillides, A., Aphamis, T., Charalambous, D. \& Ioannou, P., Modelling and simulation of transportation systems: A scenario planning approach. Automatika: Journal for Control, Measurement, Electronics, Computing \& Communications, 50, 2009.

[13] Papageorgiou, G., Damianou, P., Pitsillides, A., Aphamis, T. \& Ioannou, P., A computer simulation scenario analysis approach as a decision support tool for transportation systems planning. WIT Transactions on The Built Environment, 96, 2007.

[14] Papageorgiou, G. \& Maimaris, A., A review on current technologies for the development of intelligent pedestrian mobility systems (IPMS). 2017 IEEE 20th International Conference on Intelligent Transportation Systems (ITSC): Workshop (ITSC2017 - Workshop) - Under review, Yokohama, Japan, 2017. 